\section{THE TAI OF MUONG VAT DO NOT SPEAK THE BLACK TAI LANGUAGE}

\section{Theraphan L-Thongkum ${ }^{1}$}

\begin{abstract}
A word list of 3,343 items with Standard Thai, English and Vietnamese glosses was used for eliciting the Black Tai or Tai Dam language data at each of the twelve research sites: ten in northern Vietnam, one in northern Laos, and one in central Thaialnd. The data collected at two villages in Muong Vat could not be used for a reconstruction of Old Black Tai phonological system and a lexicon because on a phonological basis and a lexical basis, the Tai dialect of Muong Vat is not Black Tai, especially the one spoken at Ban Phat, Chieng Pan sub-district and Ban Coc Lac, Tu Nang sub-district, Son La province, Vietnam.
\end{abstract}

\section{Introduction}

The Black Tai (Tai Dam, Thai Song, Lao Song, Lao Song Dam, Phu Tai Song Dam) language has been studied by many non-Thai and Thai linguists, and by Black-Tai language specialists. (Diguet 1895, Gedney 1964, Hartmann 1985 and 1995, Don 1989, Fippinger 1970, 1971, 1975a and 1975b, Hoang Tran Nghich \& Tong Kim An 1990, Cam Cuong 1991, Witsarut Suwanwiwek 1981, Meewan Leerawat et al 1982, Chakrit Anuntrawan 1978, Wilailak

'Ph.D., Professor, Department of Linguistics, Faculty of Arts, Chulalongkorn University, Bangkok, Thailand.
Daecha 1989, Kanchana Panka 1980, Suree Pengsombat 1990, Wipawan Plungsuwan 1981, Anculee Buranasing 1988, Orapin Maneewong 1987, Kantima Wattanaprasert \& Suwattana Liamprawat 1985, Suwattana (Liamprawat) Damkham \& Kantima Wattanaprasert 1997, Oraphan Unakonsawat 1993).

In most of these previous studies, especially by Thai linguists, a Black Tai variety spoken at one location is described and in some cases compared with the other Tai or Lao dialcets spoken in nearby areas. The Black Tai varieties spoken in Laos and Vietnam have never been investigated seriously by Thai linguists. Contrarily, in the works done by non-Thai linguists, general lingusitic characteristics of the language are attempted with no emphasis on the location where it is spoken. In other words, a description and explanation of the so-called Common Black Tai is their major aim.

Not only Thai linguists but also Thai anthropologists and historians are interested in the Black Tai of Sip Song Chou Tai. Some of them believe that various features in the language and culture of the Black Tai can be taken as substantial clues for those of Southwestern Tais and the Thai (Siamese). To be able to react properly to this idea, the rasearch project "From Sip Song Chou Tai to Central Thailand: Language, Social and Cultural Changes of the Black Tai" was launched in 1997. This research project has been funded by the Princess Mahachakri Sirindhorn Research Fund, Chulalongkorn University. The sub-project on the Balck Tai language is part of this project. 
A well-known Thai anthropologist and archaeologist, Srisakara Vallibhotama (1991), and a Thai historian, Manoo Udomwet (1995), who had an opportunity to visit Muong Vat said that the Tai of Muong Vat were Black Tai from a cultural point of view. For example, their clothing was similar to the one of the Petchaburi Lao Song or Black Tai. In this paper, the author, by using linguistic evidence, would like to argue that the Tai of Muong Vat are not Black Tai in the sense that they do not speak the Black Tai Language.

\section{Language data}

To investigate the language of the Black Tai, three word lists with Standard Thai, English and Vietnamese glosses were devised and used for eliciting the language data at each of the twelve research sites: ten in northern Vietnam ${ }^{2}$ (Muong Vat 1, Muong Vat 2, Muong Lo,

\footnotetext{
${ }^{2}$ The data on Black Tai spoken at the two research sites in Laos and Thailand were collected by the author. The rest was collected by Professor Hoang Van Ma and Dr. Ta Van Thong, staff members of the linguistics Institute, the National Center for Research on Social Sciences and Humanities of Vietnam. To be certain that their collection of data was consistent with the author's standards, two Black Tai speakers studying in Hanoi were used as informants for a demonstration during the workshop. All necessary instructions, including batteries, film, blank cassettes, and so forth, were provided. At each research location, besides making recordings, they were required to photograph the village and the informants. In addition, brief information on the village where the data were collected and on the informants had to be provided. Notes on any other interesting aspects were also encouraged.
}

Muong Nam Ma, Muong La), one in northern Laos (Muong Luang Nam Tha), and one in central Thailand (Khaw Yoy district). The long word list which comprises 3,343 items and the two short additional word lists of 20 and 153 items were used for eliciting Black Tai vocabulary, tones, consonants and vowels. Every item in the short word lists was recorded on tape in order to check the phonetic transcription based on auditory judgement and for acoustical measurements to reconfirm the phonetic characteristics of tones.

Due to all types of problems, both technical and non-technical, 632 items in the long word list which were collected by the two Vietnamese linguists had to be discarded. Therefore, only 2,711 items could be used for the comparative study. The old black Tai phonological system and lexical comprising 2,300 entries, were reconstructed. In total, 1043 items in the long word list were discarded to control the quality of the data used for the analysis and reconstruction. Even though a lot of data on Black Tai can be found in other publications and many unpublished M.A. theses, they were not used because our Black Tai corpus was large enough for our research purposes. However, for a comparative and historical study, the data collected at Ban Phat, Chieng Pan sub-district and Ban Coc Lac, Tu Nang sub-district, in Muong Vat (Yen Chau) located in the province of Son La had to be excluded. On a phonological and a lexical basis, the Tai dialect of Muong Vat is not Black Tai. The detailed arguments can be found in the next section.

\section{Tai Dialect of Muong Vat}

Chamberlain (1984: 73, and 1992: 154) points out that "The Tai of Muong Vat 
(Yen Chau) in Sip Song Chou Tai speak a form of Thay Neua closer to that of Xieng Kho than to Black Tai Proper" On linguistic grounds, the author totally agree with Chamberlain that the Tai of Muong Vat, at least the two groups which live in Ban Phat and Ban Coc Lac, do not speak Black Tai. ${ }^{3}$ Chamberlain's view is based only on the pattern of tonal development: A1-234, B123-4, C123-4, DL and DS 123-4. The pattern of tone split in column A and the phonetic characteristics of the six tones in the Tai dialect of Muong Vat are not similar to those of Black Tai. The tonal analysis, the acoustical measurements of tones and the reconstruction of Old Black Tai tonal system which were done by the author support very well the idea proposed by Chamberlain, as illustrated in Figure 1.

Figure 1 Comparison of Muong Vat and Muong Lo (Black Tai) patterns of tone split

Muong Vat (Chamberlain 1984)

\begin{tabular}{|c|c|c|c|c|}
\hline A & B & C & DL & DS \\
\cline { 1 - 1 } 24 & & & & \\
\cline { 1 - 1 } 3 & \multirow{2}{*}{22} & 31 & 22 & 34 \\
\cline { 2 - 5 } & & & & \\
\hline
\end{tabular}

${ }^{3}$ Since the data on Tai Nuea spoken in Laos is not available, the author can neither support nor reject Chamberlain's claim that the Tai of Muong Vat speak a form of Tai Nuea closer to that of Xieng Kho.
Muong Vat 1 (Ban Phat)

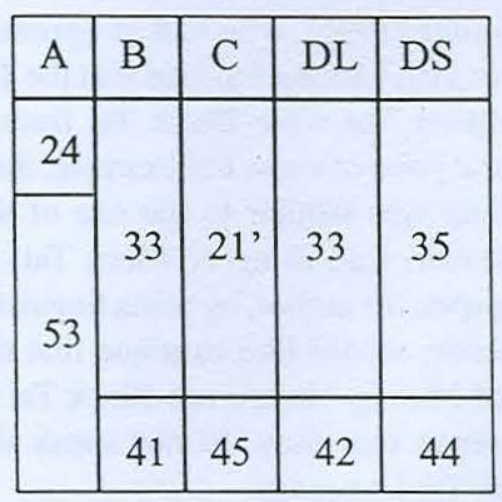

Muong Vat 2 (Ban Coc Lac)

\begin{tabular}{|c|c|c|c|c|}
\hline A & B & C & DL & DS \\
\cline { 1 - 1 } 35 & & & & \\
\cline { 1 - 1 } 4 & \multirow{2}{*}{32} & 21 & 22 & 45 \\
\cline { 2 - 5 } & & & & \\
\cline { 2 - 5 } & 42 & 44 & 32 & 34 \\
\hline
\end{tabular}

Black Tai (Muong Lo)

\begin{tabular}{|r|r|r|r|}
\hline A & B & C & DL DS \\
\hline 11 & 25 & 21 & $25 \sim 35$ \\
\hline 55 & 33 & 42 & $33 \sim 34$ \\
\hline
\end{tabular}


The patterns of sound changes as can be seen in the above examples help support the view that Tai Muong Vat is not a variety of Black Tai. Even though the speakers of Tai Muong Vat live in the area (Sonla province) which is occupied by the Black Tai people, they do speak different Tai dialect. Not only phonological differences but also lexical differences between Tai Muong Vat and Black Tai can be detected, as in the following:

\begin{tabular}{|c|c|c|}
\hline 'to call' & $\begin{array}{l}\text { i Muong Vat } \\
\text { hiək D2 }\end{array}$ & $\begin{array}{l}\text { Old Black Tai } \\
{ }^{*} \text { khe? }\end{array}$ \\
\hline 'to shoot' & jiij $\wedge 2$ & $*_{\text {ben }}$ \\
\hline $\begin{array}{l}\text { 'to crave } \\
\text { for' }\end{array}$ & zaak DLI & 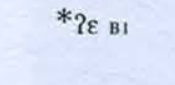 \\
\hline 'cheap' & $\mathrm{h} \varepsilon \mathrm{Al}$ & $*_{\text {thu? } \mathrm{DI}}$ \\
\hline 'gun' & thuy Bt & *?o1] в \\
\hline 'to practise' & thrp DS2 & $*_{2 \varepsilon p \mathrm{DI}}$ \\
\hline 'spear' & caw Bt & $*_{\mathrm{h} \supset ? \mathrm{D} 1}$ \\
\hline 'soaking wet' & muk DSı & $*_{\mathrm{ctrmm} \text { a2 }}$ \\
\hline 'small basket' & sa-loct & $*_{\text {SOIj } \mathrm{Cl}}$ \\
\hline 'to fan' & phr1] $A 2$ & $*_{\mathrm{vi} \sim \mathrm{bi}} \mathrm{A}_{2}$ \\
\hline 'dip-net' & vin $\mathrm{Al}$ & $*_{\mathrm{ka}}$-sa A \\
\hline
\end{tabular}

\section{Conclusion}

Even though the Black Tai living in northern Laos and central Thailand left their homeland in Sip Song Chou Tai more than two hundred years ago, they have maintained their language very well. Perhaps language is regarded by them as the most important indices of their ethnic identity. They remain proud of their origins and the old kingdom from which they were forced to flee because of wars and for political reasons. In comparison with lexical differences caused by borrowings from Vietnamese,
Regarding the Tai dialect of Muong Vat, it is reconfirmed by both phonological and lexical evidence that the one spoken at Ban Phat, Chieng Pan sub-district and Ban Coc Lac, Tu Nang subdistrict, is not Black Tai. The Tai Muong Vat dialect belongs to the $\mathrm{PH}$ language groups as the Lao language, whereas all of the Black Tai varieties spoken in Vietnam, Laos and Thailand belong to the $\mathrm{P}$ language group. The author's analysis of tone split and tone merger patterns supports Chamberlain's first claim in 1984 , based solely on his tonal analysis that the Tai of Muong Vat do not speak Black Tai. The other phonological evidence shown in this paper also help prove that our findings can not be wrong. If the historians and anthropologists still believe that the Tai of Muong Vat are Black Tai, we do need a solid proof from them. 


\section{References}

\section{In Thai}

Anchulee Buranasing. 1988. An analysis of lexical change among three generations in Thai Song dialect. Unpublished M.A. thesis, Mahidol University.

Chakrit Anuntrawan. 1978. The phonemic system of Thai Dam dialect spoken at Muban Napanat, Tambon Khao Kaeo, Amphoe Chiang Khan, Changwat Loei. Unpublished M.A. thesis, Srinakharinwirot University.

Kanchana Panka. 1980. The phonological characteristics of Lao dialects spoken in Amphoe Muang $\mathrm{N}$ akhon Pathom. Unpublished M.A. thesis, Chulalongkorn University.

Manoo Udomwet. 1995. The Tai Dam of Muong Vat. The earth magazine 2000, 2.16: 69-76.

Meewan Leerawat et al. 1982. A comparative dictionary of Bangkok Thai-Kham Müang-Tai Lue-Tai Dam. Chiangmai: Faculty of Humanities, Chiangmai University.

Orapan Unakonsawat. 1993. A comparative study of Phu Tai and Lao Song phonological s ystems. Unpublished M.A. thesis, Silpakorn University.

Sisakara Vanllibhotama. 1991. The belief in Taen of the Black Tai: The primitive religion of the Thai. Muang B oran journal, 17.3: 12-35.

Suwattana (Liamprawat) D amkham and Kantima Wattanaprasert. 1997. An analysis of lexical u se and variation among three generations in Lao language communities of the Thachin River Basin. Journal of Silpakorn University (special issue): 82-90.

Wilailuck D aecha. 1987. A comparative study of the phonology of six Tai dialects spoken in Amphoe Tha Tako, Changwat Nakhon Sawan. Unpublished M.A. thesis, Chulalongkorn Univerity.
Wisarut Suwanwiwek. 1981. Song writing system. Unpublished M.A. thesis, Silpakorn University.

\section{In English}

Cam Cuong. 1991. Black Thai language and other dialects in the system of Thai languages in Vietnam, its origin and development. Hanoi: Thai Studies Program, Hanoi University.

Chamberlain, James R. 1984. The Tai dialects of Khammouan province: Their diversity and origins. Science of language, 4: 62-95.

Chamberlain, James R. 1992. A review of historical dictionary of Laos by Martin Stuart-Fox and Mary Kooyman. Asian historical dictionaries no.6. Metuchen, N.J. and London: The Scarecrow Press Inc. Journal of the Siam Society 8 , part 1.

Don, Baccam. 1989. Tai Dam-English, English-Tai Dam vocabulary book. Eastlake, Ca: Summer Institute of Linguistics.

Diguet, Edouard. 1895. Etude de la langue tai. Hanoi.

Fippinger, Jay W. and Dorothy C. Fippinger. 1970. Black Tai phonemes with reference to White Tai. Anthropological linguistics 12.3: 8393.

Gedney, William J., Jimmy G. Harris and James R. Chamberlain (eds.), pp. 130169. Bangkok: Central Institute of English Language, Office of State University.

Gedney, William J. 1964. A comparative sketch of White, Black and Red Tai. The social science review (Bangkok), 14: 1-47.

Hartmann, John F. 1985. D ating White Thai and B lack Thai scripts. Paper presented at the $18^{\text {th }}$ International Conference on Sino-Tibetan Languages and Linguistics, organized by Ramkhamhaeng University, Bangkok, August 27-29, 1985. 
Hartmann, John F. 1995. Transmutation in a multicultural world: A comparative study of Old Lao and Tai Dam manuscript versions of the tale of prince

Hoang Tran Nghich and Tong K im An. 1990. Black Tai-Vietnamese dictionary. Hanoi: Nha Xuat Ban Khoa Hoc Xa Hoi.

Maneewong, Orapin. 1987. A comparative phonological study of Lao S ong in Petchaburi and Nakhon Pathom provinces. U npublished M.A. thesis, Mahidol University.

Pengsombat, Suree. 1990. A study of pronominal usage in spoken Tai Song. Unpublished M.A. thesis, Mahidol University.

Plungsuwan, Wipawan. 1981. A tonal comparison of Tai dialects in Ratchaburi province. Unpublished M.A. thesis, Mahidol University.

Theung or Terng. Proceedings of the International Conference on Tai Languages and Cultures, pp. 73-102. Bangkok: Thammasart University.

Note Most of the English titles of the Tai Dam materials written in Thai are provided by their authors.

\section{Acknowledgement}

I would like to thank the National Center for Research on Social Sciences and Humanities of Vietnam and the Ministry of Information and Culture of Laos PDR for their kind co-operation. Without their assistance, the investigation of Black Tai language would not have been possible. 


\section{Editor's Note:}

Comments from readers are beneficial to the author in making his/her paper clearer or more interesting. In some cases, the author may not agree with the comments and has reasons to support his/her ideas. Both the reader's comments and the author's reasons for the disagreement to the comment are educational. The editor would like to thank both Assoc. Prof. Wilaiwan Kanitthanan and Prof. Theraphan L-Thongkum for allowing us to publish their views here.

\section{Reflections on "The Tai of Muong Vat do not speak the Black Tai Language"}

1. "The Tai of Muong Vat do not speak the Black Tai language" is a contribution to the study of the development of BlackTai's phonology. The article gives the detail of tonal, consonant as well as vowel developments of four Black Tai dialects, three of Muong Vat and one of Moung Lo.

2. Based on Chamberlain's claim on Muong Vat Tai that it was more like a "Tai Nuea" than a Black Tai proper, the author set out to prove that the Tai of Ban Phat and Ban Coc Lac of Muang Vat do not speak the Black Tai language. (Without the word "proper" after Black Tai)

3 . The author proves by showing that the two Tai dialects of Muang Vat have different tonal, consonant as well as vowel developments from those of Black Tai in other places.

4. The proof is unconvincing in that we often find that subdialects of the same language can have different tonal developments. For example, Gedney reported Red Tai with three different types of tonal development as shown below.

$\begin{array}{clcc}\text { RTI } & \text { RTII } & \text { RTIII } \\ 12322 & 12352 & 12322 \\ 12322 & 42352 & 12322 \\ 12322 & 42352 & 12322 \\ 43523 & 43543 & 43521\end{array}$

(From Gedney, William. 1994. Southwestern Tai Dialects. In Thomas John Hudak (ed.) Papers on South and Southeast Asia.)

It seems that for many Tai scholars, such as Gedney, different tonal developments are not used as a criterion to group or to separate subdialects. Gedney also reported different languages with the same type of tonal development, for example, Black Tai of Son La and White Tai from Lai Chao.

\begin{tabular}{|c|c|}
\hline Black Tai & White Tai \\
\hline 12322 & 12322 \\
\hline 12322 & 1232 \\
\hline 12322 & 1232 \\
\hline 45655 & 4564 \\
\hline
\end{tabular}

Both groups show development from Proto-Tai *b *d *g to “p-, t-, k-".

5. Areal features have an important role in language development in North Vietnam. North Vietnam is a great show case of areal linguistics with 54 tribes. Languages of different language families share a lot of common vocabulary. Languages of the same language family may use different vocabulary if they are far apart and on the contrary,different Tai languages, i.e. Tay and Nung, can have many features in common. Tone $\mathrm{CI}$ in most Tai and 


\section{Answers to Dr. Wilaiwan Khanittanan's reflections}

1. The author would like to reconfirm that the varieties of Black Tai which the author came across in available Black Tai dictionaries, research reports and in her linguistic field trips had the same type of tone system and tonal development, i.e., six tones (A1, A2, B1, B2, C1, C2, $\mathrm{D} 1=\mathrm{B} 1, \mathrm{D} 2=\mathrm{B} 2)$. The differences found were phonetic characteristics of the six tones and the variation of few tones in one or two dialects which could have been caused by language contact, such as tone $\mathrm{A} 1$ in the Black Tai varieties of Chiang K han (Loei) and Khaw Yoy (Phetchaburi).

The pattern of tonal development and the phonetic characteristics of tones in fifteen Black Tai dialects or varieties are shown below.

Pattern of tonal development

\begin{tabular}{|c|c|c|c|c|}
\hline $\begin{array}{c}\text { PT } \\
\text { Tones }\end{array}$ & ${ }^{*} \mathbf{A}$ & ${ }^{*} \mathbf{B}$ & ${ }^{*} \mathbf{C}$ & ${ }^{*} \mathbf{D}$ \\
\hline $\mathbf{1 / 1 - 3}$ & $\mathrm{T} 1$ & $\mathrm{~T} 3$ & $\mathrm{~T} 5$ & T3 \\
\hline $\mathbf{2 / 4}$ & $\mathrm{T} 2$ & $\mathrm{~T} 4$ & $\mathrm{~T} 6$ & $\mathrm{~T} 4$ \\
\hline \multicolumn{5}{|c}{} \\
\hline
\end{tabular}

Phonetic characteristics of the tones in non-checked syllables

\begin{tabular}{|c|c|c|c|c|c|c|}
\hline Location & $\begin{array}{l}\text { Tone1 } \\
\text { (A1) }\end{array}$ & $\begin{array}{l}\text { Tone2 } \\
\text { (A2) }\end{array}$ & $\begin{array}{l}\text { Tone3 } \\
\text { (B1) }\end{array}$ & $\begin{array}{l}\text { Tone4 } \\
\text { (B2) }\end{array}$ & $\begin{array}{l}\text { Tone5 } \\
\text { (C1) }\end{array}$ & $\begin{array}{l}\text { Tone6 } \\
\text { (C2) }\end{array}$ \\
\hline \multicolumn{7}{|l|}{ Vietnam } \\
\hline M.Lo & 11 & 55 & 25 & 33 & 21 & 42 \\
\hline M.Mua & 22 & 44 & 24 & 33 & 21 & 32 \\
\hline M.Than & 22 & 55 & 25 & 44 & $21 \sim 12$ & 43 \\
\hline M.Thanh & 22 & 44 & 35 & 33 & 21 & 41 \\
\hline M.Kway & 11 & 44 & 24 & 33 & 21 & $4 \hat{2}$ \\
\hline M.Muay & 22 & 44 & 24 & 33 & 21 & 42 \\
\hline M.Nam Ma & 22 & 44 & 25 & 33 & 21 & 31 \\
\hline M.La & 33 & 55 & 34 & 44 & 21 & 31 \\
\hline \multicolumn{7}{|l|}{ Laos } \\
\hline $\begin{array}{l}\text { M.Luang } \\
\text { Nam Tha }\end{array}$ & 22 & 55 & 35 & 33 & $31^{\prime}$ & $42^{\prime}$ \\
\hline M.Nam Bak & 22 & 454 & 24 & 33 & $31^{\prime}$ & $453^{\prime}$ \\
\hline M.May & 22 & 44 & 24 & 33 & $31^{\prime}$ & $42^{\prime}$ \\
\hline \multicolumn{7}{|l|}{ Thailand } \\
\hline A.Khaw Yoy & $22 \sim 33$ & 55 & 35 & 33 & $31^{\prime}$ & 453 \\
\hline $\begin{array}{l}\text { A.Chiang } \\
\text { Khan }\end{array}$ & $332 \sim 343$ & 454 & 35 & 33 & $31^{\prime}$ & 352 \\
\hline $\begin{array}{l}\text { A.Muong } \\
\text { (N.Prathom) }\end{array}$ & 24 & 354 & 35 & 44 & 33 & 43 \\
\hline $\begin{array}{l}\text { A.Tha Tako } \\
\text { (N.Sawan) }\end{array}$ & 14 & 343 & 35 & 33 & $22^{\prime}$ & 42 \\
\hline
\end{tabular}


Besides Black Tai, the author also had an opportunity to work with the speakers of Red Tai living in Vietnam and Laos at four locations. The typical pattern of tone split and merger in Red Tai can be summarized and illustrated as below.

Pattern of tonal development

\begin{tabular}{|c|c|c|c|c|c|}
\hline PT Tones & $* \mathbf{A}$ & $* \mathbf{B}$ & ${ }^{*} \mathbf{C}$ & *DL & *DS \\
\hline $\mathbf{1 / 1 - 3}$ & $\mathrm{T} 1$ & $\mathrm{~T} 3$ & $\mathrm{~T} 4$ & $\mathrm{~T} 3$ & $\begin{array}{c}\mathrm{T} 2 / \mathrm{T} 5 / \mathrm{T} 2 / \\
\mathrm{T} 3\end{array}$ \\
\hline $\mathbf{2 / 4}$ & $\mathrm{T} 2$ & $\mathrm{~T} 4$ & $\mathrm{~T} 5$ & $\mathrm{~T} 4$ & $\begin{array}{c}\mathrm{T} 1 / \mathrm{T} 3 / \mathrm{T} 5 / \\
\mathrm{T} 2\end{array}$ \\
\hline
\end{tabular}

Phonetic characteristics of the tones in non-checked syllables

\begin{tabular}{|l|c|c|c|c|c|}
\hline \multicolumn{1}{|c|}{ Location } & $\begin{array}{c}\text { Tone1 } \\
\text { (A1) }\end{array}$ & $\begin{array}{c}\text { Tone2 } \\
(\mathbf{A 2})\end{array}$ & $\begin{array}{c}\text { Tone 3 } \\
\text { (B1) }\end{array}$ & $\begin{array}{c}\text { Tone4 } \\
\text { (B2\&C1) }\end{array}$ & $\begin{array}{c}\text { Tone5 } \\
\text { (C2) }\end{array}$ \\
\hline $\begin{array}{l}\text { Mai Ha } \\
\text { (Mai Chau) }\end{array}$ & 33 & 22 & 35 & $13^{\prime}$ & 41 \\
\hline $\begin{array}{l}\text { Bao La } \\
\text { (Mai Chao) }\end{array}$ & 454 & 342 & 44 & 35 & 31 \\
\hline $\begin{array}{l}\text { Phon Hom } \\
\text { (Nam Bak) }\end{array}$ & 232 & 343 & 44 & 24 & 31 \\
\hline $\begin{array}{l}\text { Nong Bua Thong } \\
\text { (Sikhottabong) }\end{array}$ & 343 & 22 & 33 & 24 & 44 \\
\hline
\end{tabular}

The data given above confirm that in non-checked or live syllables, the Red Tai language has five tones with the merger of $\mathrm{B} 2 / \mathrm{B} 4$ tone to $\mathrm{C} 1 / \mathrm{C} 1-3$ tone. The diverse behavior of tones DS1/DS13 and DS2/DS4 in Red Tai can be used as a criterion for classifying and subgrouping the Red Tai language into different varieties.

Even though the number of Red Tai speakers is less than that of Black Tai, there can be more varieties of Red Tai due to language contact. Language diversity in the areas where the speakers of Red Tai inhabit has reinforced the situation. In other words, small number of speakers and the diverse nature of linguistic areas are the major causes of Red Tai varieties. In Vietnam and Laos, being Black Tai seems to be more prestigious than being White Tai, Red Tai or the other Tai ethnic groups. The Black Tai have more power and more roles to play at national level, especially in administration and politics in the two countries. The feeling of superiority makes them feel proud and try to maintain their ethnicity and language. For Red Tai and other small Tai ethnic groups, it is natural that they would like to be affiliated with the Black Tai since it is a way to raise their social status. 
Regarding White Tai, the author has also worked on some White Tai varieties spoken in Vietnam and Laos. Certainly, the pattern of tonal development of White Tai (WT) and Black Tai (BT) are the same as illustrated below.

\section{Black Tai}

\begin{tabular}{|c|c|c|c|c|}
\hline PT Tones & $* \mathbf{A}$ & $\boldsymbol{* B}_{\mathbf{B}}$ & $* \mathbf{C}$ & $* \mathbf{D}$ \\
\hline $\mathbf{1 / 1 - 3}$ & $\mathrm{T} 1$ & $\mathrm{~T} 3$ & $\mathrm{~T} 5$ & T3 \\
\hline $\mathbf{2 / 4}$ & $\mathrm{T} 2$ & $\mathrm{~T} 4$ & $\mathrm{~T} 6$ & T4 \\
\hline
\end{tabular}

White Tai

\begin{tabular}{|c|c|c|c|c|}
\hline PT Tones & $* \mathrm{~A}$ & $* \mathrm{~B}$ & $* \mathrm{C}$ & $* \mathrm{D}$ \\
\hline $1 / 1-3$ & T1 & T3 & T5 & T3 \\
\hline $2 / 4$ & $\mathrm{~T} 2$ & T4 & T6 & T4 \\
\hline
\end{tabular}

Phonetic characteristics of the six White Tai tones (non-checked syllable)

\begin{tabular}{|c|c|c|c|c|c|c|}
\hline Location & $\begin{array}{c}\text { Tone1 } \\
\text { (A1) }\end{array}$ & $\begin{array}{c}\text { Tone2 } \\
(\mathbf{A 2})\end{array}$ & $\begin{array}{c}\text { Tone3 } \\
\text { (B1) }\end{array}$ & $\begin{array}{c}\text { Tone4 } \\
(\mathbf{B 2})\end{array}$ & $\begin{array}{c}\text { Tone5 } \\
\text { (C1) }\end{array}$ & $\begin{array}{c}\text { Tone6 } \\
\text { (C2) }\end{array}$ \\
\hline $\begin{array}{c}\text { M.So } \\
\text { (Lai Cau) }\end{array}$ & 22 & $453^{\prime}$ & 35 & 44 & 213 & 41 \\
\hline $\begin{array}{c}\text { M.Baeng } \\
\text { (Oudomxi) }\end{array}$ & 22 & $44^{\prime}$ & 33 & 452 & 34 & 41 \\
\hline
\end{tabular}

However the development of diphthongs *iə, *uə, *uə and *aur differ as shown below.

\begin{tabular}{|c|c|c|}
\hline Proto-Tai & Black-tai & White Tai \\
\hline$*$ iə & iə & e \\
\hline *uə & uə & $\gamma$ \\
\hline$*$ uə & uə & o \\
\hline *aur & aur & aur \\
\hline
\end{tabular}

Regarding the development of diphthongs, Black Tai and White Tai are distinct. 